\title{
Potential Tasks and Research Issues for Mobile Robots in RoboCup Rescue
}

\author{
Robin R. Murphy, Jenn Casper, Mark Micire ${ }^{1}$ \\ Computer Science and Engineering \\ University of South Florida, Tampa FL 33620, USA
}

\begin{abstract}
Previous work[5] has summarized our experiences working with the Hillsborough Fire Rescue Department and FEMA documents pertaining to Urban Search and Rescue. This paper discusses the lessons learned and casts them into four main categories of tasks for the physical agent portion of RoboCup-Rescue: 1) reconnaissance and site assessment, 2) rescuer safety, 3) victim detection, and 4) mapping and characterizing the structure.
\end{abstract}

\section{Introduction}

As chronicled in [6], we have been researching mobile robots for Urban Search and Rescue (USAR) since 1996. Our hands-on experience includes having a graduate student and thesis based on the Oklahoma City bombing [4] and attending training classes in USAR with the Hillsborough County Fire Rescue department. Previous work [5] has investigated the US FEMA (Federal Emergency Management Agency) documents concerning USAR in order to generate an understanding of the potential roles of intelligent mobile robots. This understanding, or domain knowledge, should help RoboCup Rescue provide a meaningful competition and to stimulate research which will have practical applications. This paper reviews and duplicates salient portions of [5].

Fire rescue departments in the United States are responsible for responding to four different USAR scenarios, each with its own requirements: HAZMAT, bomb threats, collapsed buildings, and trench rescue. Collapsed buildings and trench rescue share many similarities (see Fig. 1). In both cases, victims may be buried and require search and detection. When found, most often the victim cannot accurately describe where his/her limbs are located due to dis-orientation, stress, etc., interfering with the extrication effort. The victims may provide incorrect information that leads to further injury as rescuers remove rubble. In the case of a completely buried victim, rescuers depend upon witnesses and communicating with other victims in order to determine the location of other victims. Equipment used in these types of USAR efforts must be weather proof and intrinsically safe, in order to both operate under all conditions in the rubble and to withstand being washed down thoroughly if any hazardous materials or body fluids are encountered.

Collapsed buildings need small, highly mobile platforms. Current technical rescue operations can mechanically penetrate 18 feet into a collapsed structure 

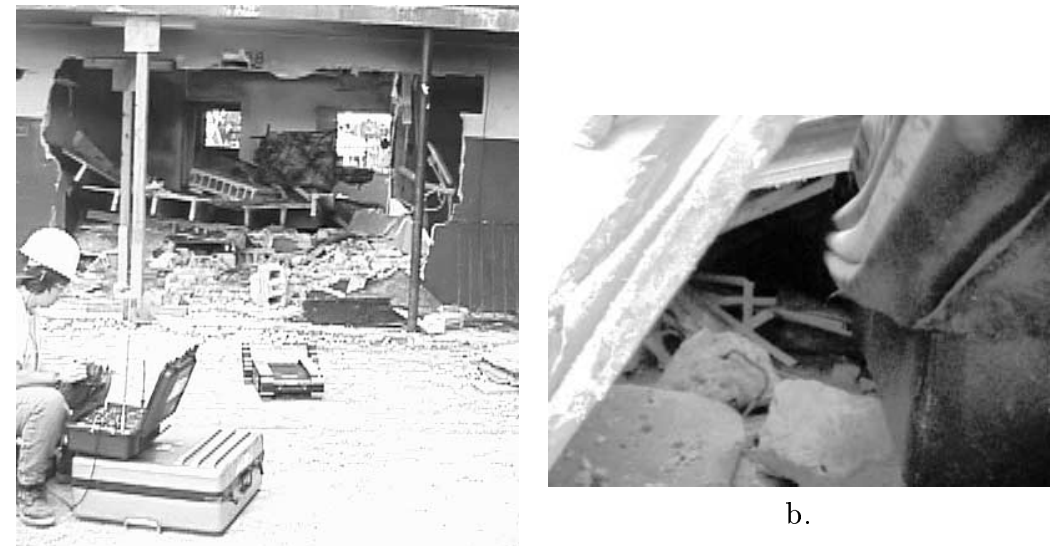

b.

a.

Fig. 1. a.) Testing a mobile robot at the SRDR USAR test site, and b.) a typical void formed by rubble falling against furniture.

that is un-enterable by humans. This is accomplished using a borescope to provide a video feed to the rescuers. The designer must also make the robot rugged, as it might fall or become trapped in such a way that a weaker robot would be crushed or damaged. The robot must also be self-rightable in that it will not be immobilized by being turned over. It is likely to encounter ground which will dramatically alter the robot's orientation and this must be accounted for.

\section{Phases in Collapsed Building Rescues}

In order to understand how the physical agent component of RoboCup-Rescue can be of use to rescue workers, it is helpful to review a rescue scenario. Collapsed building rescue efforts follow five phases. First, the incident command system for coordinating the rescuers and controlling the media and family is established and an initial reconnaissance is performed. Though the actual time depends on the magnitude of the incident, the average set up time is around three hours.

The second phase is to rescue and recover surface victims. Surface victims are people who have been hit with flying debris, or injured during during a fall. They are the victims that have the best chance of surviving because they are easily reached and removed from further harm. Often surface victims are assisted by other witnesses or civilians before the rescue teams arrive on the scene. Surface victims account for about $50 \%$ of the total number of victims recovered. Despite the likelihood of surface victims, it is important not to approach the incident area before the site is assessed for safe entry, as secondary collapses and aftershocks immediately threaten impatient rescuers and trapped victims. 
The third phase is the location and extrication of lightly trapped victims. Lightly trapped victims are those that can be removed by one or two rescuers, usually by removing a piece of furniture or light rubble. Lightly trapped victims account for approximately $30 \%$ of the total number of victims recovered. Victims are currently located using search dogs, listening devices, thermal cameras, etc.

The fourth phase, search of void spaces, requires trained and specially equipped rescuers working in conjunction with structural engineers. Approximately $15 \%$ of the total number of victims are found in void spaces. These victims are trapped in a void and are unable to move. The average removal time is 4 hours with 10 highly trained and equipped rescuers. Approximately $5 \%$ of the total number of victims are entombed. Entombed means to be trapped by main building components, such as a wall or support beam. The delicacy with which a support beam or wall needs to be removed results in an approximate victim rescue time of 10 hours. Sensors currently used to locate victims in void spaces include thermal imaging cameras, directional listening devices, fiber optic cameras (borescopes), etc.

The fifth phase is recovery and cleaning up the incident area. Recovery is different from rescue in that it is assumed that no one is left living and the rescuers are now removing bodies. Heavy equipment is used to move remove rubble. However, there is a small chance that a live victim may be found, so removal teams must be cautious.

\section{Tasks for RoboCup-Rescue Physical Agents}

Earlier discussions of the physical agent league for RoboCup have discussed both victim detection and extrication tasks. This poster discusses only the subtasks in victim detection. Victim detection is the first step before a victim can be extricated, also extrication is hardware intensive while much of detection requires advances in artificial intelligence and software.

\subsection{Reconnaissance and Site Assessment Subtask}

One potential role of mobile robots is to provide an initial survey of the site, without risking rescuers or triggering a further collapse. The building, or hot zone, must be assumed to be structurally unsound until a qualified civil engineer can inspect it. The weight and impact of humans walking around, climbing, or setting up equipment could trigger a further collapse.

Generally, it takes three hours for the rescue team to set up its incident command structure and to do an outer circle check. Lightweight mobile robots could be deployed immediately to do an outer circle check while the human team was setting up its incident command system and establishing the hot, warm, and cold zones. This would allow the incident commander and relevant personnel to have an accurate, metric layout of the structure. The robot(s) and supporting AI may be able to identify and rank possible voids for entry into the structure. 


\begin{tabular}{|l|l|}
\hline 30 minutes & $91 \%$ survive \\
\hline 1 day & $81 \%$ survive \\
\hline 2 days & $36.7 \%$ survive \\
\hline 3 days & $33.7 \%$ survive \\
\hline 4 days & $19 \%$ survive \\
\hline 5 days & $7.4 \%$ survive \\
\hline
\end{tabular}

Table 1. Trapped victim survival rate.

\subsection{Rescuer Safety Subtask}

Intelligent robots are also needed for the humanitarian reason of saving the lives of victims and rescuers. In the 1985 Mexico City earthquake, 135 rescuers died. 65 of these deaths were due to drowning when ground water flooded the confined space area where they were searching for earthquake victims. In order to minimize potential injuries and problems, hazards need to be recognized and eliminated. There are seven major hazard categories: structural instability, overhead, surface, and below-grad hazards, leaking utilities, hazardous materials, and incidental hazards. A structural instability hazard could be a weakened floor. Ceiling wires threaten entanglement and are an example of overhead hazards. Surface hazards include debris laying on the ground, such as glass or nails. This threatens the rescuers as well as the rescue dogs. An example of a below-grade hazard is a broken gas pipe in a basement. Rescuers could potentially fall into the basement which might lack oxygen. Utilities running to the site could cause an explosion. Incident hazards include fire and smoke.

A robot could potentially provide the capability to recognize hazards, going into voids before humans. The robot would have to carry a sensor suite suitable for monitoring, which is quite different from navigation and victim detection.

\subsection{Victim Detection Subtask}

The aim of a rescue is to reduce the amount of time a victim is entrapped. Table 1 displays results of research conducted after the 1988 Armenian Earthquake[1]. The percentage of survivors is directly related to the entrapment time.

There are several ways of detecting victims used by rescuers today. The most common sensors are TV cameras, digital thermal cameras, and acoustic probes. Human rescuers most commonly use vision. While this might seem intuitively obvious, there are several facets that make this particularly difficult for robotics. First, traditional color segmentation and region detection will most likely fail because when victims are trapped within a collapsed building, they are typically covered with dust and dirt, camouflaging them within their environment. Movement would be another good visual detector to augment the color deficiencies, but because the victims are very often unconscious, this does not completely resolve the vision issues. Texture recognition may be another possible solution, but 


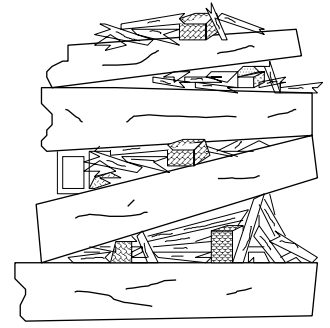

a.

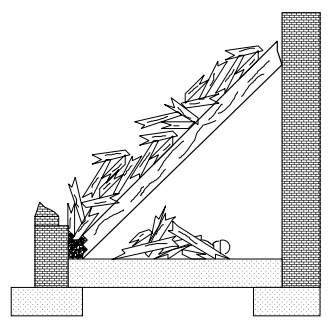

b.

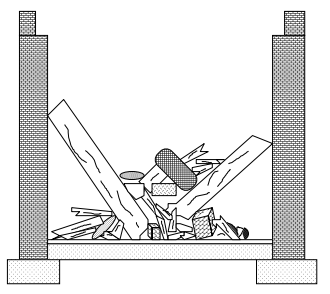

c.

Fig. 2. Three types of collapsed building structures: a. pancake, b. lean-to, c. v-shape.

clearly much more research must be made in the area of visual victim recognition if it is to be feasible in this domain. Thermal imaging cameras have recently increased in accuracy and decreased in price as to make this an extremely useful tool for locating victims. In smoke or dust filled rooms, these temperature sensitive cameras can provide almost x-ray vision for a robot completely blind within the visible spectrum. Even lightly buried victims have a heat signature that stands out from the environment. Using adapted versions of classical image processing algorithms, false color pictures could quickly be analyzed for victim identification.

\subsection{Mapping and Characterizing Structure Subtask}

It should be clear from the previous tasks that any information about the layout of the structure and the nature of the collapse will help rescuers determine what resources should be deployed. The type of voids in the structure can also help determine where to look to survivors and how to plan to extricate them.

Different types of voids are created from the collapse mechanism. The three types of collapses are: pancake, lean-to, and $\mathrm{v}$ shape. A pancake collapse is prevalent with multi-story buildings and results in a cement slab stack. Entire building floors are reduced to inches in most cases. Often these voids are large enough to server as a survivable space for victims, but are confined and difficult to access. In a pancake structure, as shown in Figure 2a, the spaces between the cement slabs needs to be searched for victims. A lean-to collapse is typically the result of a wall failure causing the floor or ceiling to fall on one end while being supported at the other end by the standing wall. The triangular shaped void serves as a survivable void for victims. A v shape collapse occurs when the center weight of a floor or ceiling is too much that the floor collapses in the middle.

\section{Conclusions}

Physical agents should be an important part of RoboCup-Rescue because of the potential impact of robots, especially in victim detection types of tasks. Vic- 
tim detection in USAR poses four major subtasks for intelligent mobile robots: reconnaissance and assessment, rescuer safety, victim detection, and mapping. Victim detection and mapping are two tasks which are more amenable to being simulated in a competition venue. The tasks are on the "inside" of the hot zone, encouraging the development of platforms and navigation algorithms suiting for operating in confined spaces. The tasks are also amenable to automation, since it currently takes ten specially trained researchers four hours to find and remove one victim trapped in a void space and ten hours to find and remove one entombed victim. These two categories of victims account for $20 \%$ of the total victims in a building collapse. Given that the survival rate of victims drops to $33.7 \%$ after 48 hours, anything that speeds up the rescue of trapped victims will be helpful.

A testbed for physical agents should encourage the development and meaningful evaluation of the intelligence of the agents, as well as platform suitability. In particular, the league should assume that rescue workers are likely to have heterogeneous agents, with differing mobility, sensing, computation, and physical abilities. The testbed should provide opportunities to exercise organic sensing and behaviors needed for navigation in confined spaces as well as for mission sensing needed for victim detection. Also, the testbed should reflect the same percentage distribution of victims in different parts of the collapsed structure (on the surface, lightly trapped, entombed). Finally, the competition should reward the integration of software with hardware, rather than just hardware alone due to the difficulties of teleoperating robots.

\section{Acknowledgments}

The authors would like to thank Special Operations Chief, Ronald Rogers, and Jeff Hewitt of the Hillsborough County Fire Rescue, and LTC John Blitch for their helpful discussions. Some photographs were collected during constructive experiments funded under the DARPA TMR program at the SRDR USAR training site in Miami Beach.

\section{References}

1. Rescue Systems 1. National Fire Academy, 1993.

2. Technical Rescue Program Development Manual. United States Fire Administration, 1996.

3. Standard on Operations and Training for Technical Rescue Incidents. National Fire Protection Association, 1999.

4. J.G. Blitch. Knobsar: An expert system prototype for robot assisted urban search and rescue. Masters Thesis, 1996.

5. J. Casper and R.R. Murphy. Issues in intelligent robots for search and rescue. In SPIE Ground Vehicle Technology II, 2000.

6. R.R. Murphy. Marsupial robots for urban search and rescue. IEEE Intelligent Systems, 15(2):14-19, 2000. 